\title{
A novel SRY missense mutation affecting nuclear import in a 46, XY female patient with bilateral gonadoblastoma
}

Remko Hersmus ${ }^{1}$, Bertie HCGM de Leeuw ${ }^{1}$, Hans Stoop ${ }^{1}$, Pascal Bernard ${ }^{2}$, Helena C van Doorn ${ }^{3}$, Hennie T Brüggenwirth ${ }^{4}$, Stenvert LS Drop ${ }^{5}$, J Wolter Oosterhuis ${ }^{1}$, Vincent R Harley ${ }^{2}$ and Leendert HJ Looijenga*1

\footnotetext{
${ }^{1}$ Department of Pathology, Erasmus MC-University Medical Center Rotterdam, Josephine Nefkens Institute, Daniel den Hoed Cancer Center, Rotterdam, The Netherlands; ${ }^{2}$ Human Molecular Genetics Laboratory, Prince Henry's Institute of Medical Research, Clayton, Victoria, Australia; ${ }^{3}$ Department of Gynaecologic Oncology, Erasmus MC-University Medical Center Rotterdam, Rotterdam, The Netherlands; ${ }^{4}$ Department of Clinical Genetics, Erasmus MC-University Medical Center Rotterdam, Rotterdam, The Netherlands and ${ }^{5}$ Department of Pediatric Endocrinology, Erasmus MC-University Medical Center Rotterdam, Sophia, Rotterdam, The Netherlands
}

Patients with disorders of sex development (DSD), especially those with gonadal dysgenesis and hypovirilization, are at risk of developing the so-called type II germ cell tumors (GCTs). Both carcinoma in situ and gonadoblastoma (GB) can be the precursor lesion, resulting in a seminomatous or nonseminomatous invasive cancer. $S R Y$ mutations residing in the HMG domain are found in $10-15 \%$ of $46, X Y$ gonadal dysgenesis cases. This domain contains two nuclear localization signals (NLSs). In this study, we report a unique case of a phenotypical normal woman, diagnosed as a patient with $46, \mathrm{XY}$ gonadal dysgenesis, with an NLS missense mutation, on the basis of the histological diagnosis of a unilateral GB. The normal role of SRY in gonadal development is the upregulation of SOX9 expression. The premalignant lesion of the initially removed gonad was positive for OCT3/4, TSPY and stem cell factor in germ cells, and for FOXL2 in the stromal component (ie, granulosa cells), but not for SOX9. On the basis of these findings, prophylactical gonadectomy of the other gonad was performed, also showing a GB lesion positive for both FOXL2 (ovary) and SOX9 (testis). The identified W70L mutation in the SRY gene resulted in a 50\% reduction in the nuclear accumulation of the mutant protein compared with wild type. This likely explains the diminished SOX9 expression, and therefore the lack of proper Sertoli cell differentiation during development. This case shows the value of the proper diagnosis of human GCTs in identification of patients with DSD, which allows subsequent early diagnosis and prevention of the development of an invasive cancer, likely to be treated by chemotherapy at young age.

European Journal of Human Genetics (2009) 17, 1642-1649; doi:10.1038/ejhg.2009.96; published online 10 June 2009

Keywords: disorders of sex development; germ cell tumors; gonadoblastoma; SRY

\footnotetext{
*Correspondence: Professor LHJ Looijenga, Department of Pathology, Erasmus MC-University Medical Center Rotterdam, Josephine Nefkens Institute, Building Be, Room 430b, PO Box 2040, Rotterdam, 3000 CA, The Netherlands.

Tel: + 3110704 4329; Fax: + 31107044365

E-mail: I.looijenga@erasmusmc.nl

Received 20 February 2009; revised 15 April 2009; accepted 7 May 2009; published online 10 June 2009
}

Introduction

Congenital conditions in which the development of chromosomal, gonadal or anatomical sex is atypical are referred to as disorders of sex development (DSD), previously known as intersex. ${ }^{1}$ DSD patients with $46, \mathrm{XY}$ complete gonadal dysgenesis (previously referred to as 
Swyer's syndrome) have a high risk of developing a type II malignant germ cell tumor (GCT) with gonadoblastoma (GB), often bilateral, as the precursor lesion (see Cools et $a l^{2}$ for a review). What these patients have in common is that during early embryonic development, the initially immature bi-potential gonads fail to differentiate along the male (testicular) pathway. Pivotal to this process is the sex-determining region on the Y chromosome (SRY) gene. Typically during male development, expression of SRY in the gonadal stromal cells will upregulate the transcription of another key DSD gene and transcription factor SOX9 through DNA binding to the testis enhancer of SOX9.,4,5 This will subsequently lead to a cascade of signal transduction with differentiation of the stromal cells into (pre-) Sertoli cells. The development of functional Sertoli cells produces anti-Müllerian Hormone $(\mathrm{AMH})$ and directs the formation of testosterone-producing Leydig cells, ultimately giving rise to male internal and external genitalia. ${ }^{6}$ In $10-$ $15 \%$ of XY patients with male-to-female sex reversal $(46, X Y$ DSD), inactivating mutations in $S R Y$ have been identified, with the majority residing in the HMG domain, and affecting the binding and bending of DNA. ${ }^{7,8}$ At the Nand C-terminal ends of this domain, two nuclear localization signals (NLSs) are present. The biological effect of a number of the reported mutations can be explained by the fact that they reside in one of these NLSs, thereby disrupting nuclear import. In general, mutations in $S R Y$ can lead to the failure of the bi-potential gonads to develop into testes with the consequence that testosterone and AMH will not be produced. Without testosterone, the external genitalia will not virilize, and the Wolffian ducts fail to develop into epididymis, vas deferens and seminal vesicles. Without $\mathrm{AMH}$, the Müllerian ducts will not regress, and subsequently develop into female internal reproductive organs, namely the fallopian tubes, uterus and the upper portion of the vagina. ${ }^{6}$ At birth, these patients can show a phenotypical female appearance, but they may present at puberty with the absence of secondary sexual characteristics and primary amenorrhea. This is due to complete gonadal dysgenesis and lack of ovarian function. Therefore, it is unusual to observe a GB in a presumed normal female after puberty.

GB consists of a mixture of embryonic germ cells (primordial germ cells/gonocytes) and supportive cells that resemble immature Sertoli/granulosa cells. ${ }^{9}$ It is recognized as the in situ germ cell malignancy, which in a substantial number of cases will develop into an invasive tumor (dysgerminoma or, less frequently, non-dysgerminoma). Carcinoma in situ (CIS), the precursor of the seminoma and non-seminomatous tumors in the testis, is the GB counterpart of the male gonad. It exists in close association with Sertoli cells. ${ }^{10}$ The supportive cells in GB stain positive for the granulosa cell marker, FOXL2, and are (almost) always negative for the Sertoli cell marker, SOX9. ${ }^{11}$ This reflects a blockage in testicular differentiation. CIS, GB and seminoma, dysgerminoma and embryonal carcinoma are posi- tive for OCT3/4, which is a reliable marker for type II GCTs with pluripotent potential. ${ }^{12-14}$ OCT3/4 is also known as POU5F1, and is one of the transcription factors involved in the regulation of pluripotency. ${ }^{14-16}$ In normal development, OCT3/4 is specifically found in primordial germ cells/gonocytes, but is absent at later developmental stages. In cases of maturation delay, as can be observed in DSD patients with hypovirilization and gonadal dysgenesis, this can lead to an overdiagnosis. ${ }^{17}$ Recently it was shown that stem cell factor (SCF) is an additional valuable marker, which distinguishes germ cells delayed in their maturation from malignant germ cells, being negative and positive, respectively. ${ }^{18}$ The development of a GB is dependent on the presence of part of the $\mathrm{Y}$ chromosome, known as the GBY region. ${ }^{19}$ One of the putative candidate genes for the involvement of this region is TSPY, which denotes Testis Specific Protein on the Y chromosome. ${ }^{20}$ The encoded protein is found to be highly expressed in CIS and GB. ${ }^{21}$

In this study, we report a unique case of $\mathrm{GB}$ in a phenotypical adult woman of 26 years of age. In the DNA extracted from peripheral blood cells of this patient, a novel mutation was identified in the N-terminal NLS of SRY, resulting in reduced nuclear import, leading to gonadal dysgenesis. This was identified on the basis of the diagnosis of a unilateral GB. Subsequent prophylatic removal of the remaining gonad indeed showed the presence of another GB. This intervention prevented the development of an invasive type II GCT and the need of chemotherapy for treatment.

\section{Materials and methods \\ Immunohistochemical staining}

Immunohistochemistry was performed on paraffin-embedded slides of $3-\mu \mathrm{m}$ thickness. The antibodies used directed against OCT3/4, SCF, TSPY, SOX9 and FOXL2 have been described before. ${ }^{11,13,18,22,23}$ Briefly, after deparaffinization and $5 \mathrm{~min}$ incubation in $3 \% \mathrm{H}_{2} \mathrm{O}_{2}$ for inactivating endogenous peroxidase activity, antigen retrieval was carried out by heating under pressure of up to $1.2 \mathrm{bar}$ in an appropriate buffer. After blocking endogenous biotin using the Avidin/Biotin Blocking Kit (SP-2001; Vector Laboratories, Burlingame, CA, USA), sections were incubated either overnight at $4{ }^{\circ} \mathrm{C}$ (SCF, TSPY) or for $2 \mathrm{~h}$ at room temperature (OCT3/4, SOX9, FOXL2) and detected using the appropriate biotinylated secondary antibodies and visualized using the avidin-biotin detection and substrate kits (Vector Laboratories). Double staining for FOXL2 and SOX9 was carried out as described before. ${ }^{11}$

\section{Fluorescent in situ hybridization}

Slides of $5-\mu \mathrm{m}$ thickness were deparaffinized and heated under pressure as described above. The slides were digested using 0.01\% Pepsin (Sigma Aldrich, St Louis, MO, USA) in 
$0.02 \mathrm{M}$ of $\mathrm{HCl}$ at $37^{\circ} \mathrm{C}$, with an optimal digestion time of $2.5 \mathrm{~min}$. The slides were rinsed, dehydrated and the probes dissolved in a hybridization mixture were applied. Probes for centromere X (BamHI) and centromere Y (DYZ3) were used and labeled with digoxigenin-11-dUTP and biotin16-dUTP (Roche Diagnostics, Mannheim, Germany) using a Nick-Translation Kit (Gibco BRL, Paisley, UK). After denaturation $\left(80^{\circ} \mathrm{C}\right.$ for $\left.10 \mathrm{~min}\right)$, overnight hybridization $\left(37^{\circ} \mathrm{C}\right)$ and washing steps, probes were visualized using Cy3-conjugated avidin (1:100, Jackson ImmunoResearch, West Grove, PA, USA) and Sheep-anti-dig FITC (1:50, Roche Diagnostics) and analyzed using a fluorescent microscope (Leica Microsystems, Rijswijk, The Netherlands).

\section{SRY functional study and plasmids}

Direct sequencing of the $S R Y$ gene on peripheral blood DNA from the patient was performed at the Department of Clinical Genetics.

pcDNA3-FLAG-SRY and pcDNA3-FLAG-R75N plasmids have been previously described. ${ }^{24}$ pcDNA3-FLAG-W70L was created by PCR with the primer sequence, 5'-CATCATG GATCCGCCACCATGGACTACAAAGACGATGACGACAAGA TGCAATCTTAT-3' containing a BamHI restriction site, KOZAK translational start site, FLAG-tag epitope, SRY ATG site and 5'-ATGATGAATTCTACAGCTTTGTCCAG-3' containing a EcoRI restriction site and the SRY TAG site using standard conditions. The PCR product was subsequently cloned into the pcDNA3 vector using the introduced restriction sites. All constructs were verified by sequencing.

NT2/D1 cells seeded in six-well plates were transfected with $2 \mu \mathrm{g}$ per well of pcDNA3-FLAG-SRY wild type, the pcDNA3-FLAG-R75N mutant or the pcDNA3-FLAG-W70L mutant using Fugene 6 (Roche Diagnostics). After transfection, immunohistochemistry was carried out using mouse monoclonal antibody against the FLAG tag (1:400). The secondary antibody used was Alexa 488-conjugated donkey anti-mouse IgG (1:500, Molecular Probes, Eugene, OR, USA). DNA was stained with $0.1 \mu \mathrm{g} / \mathrm{ml}$ of $4^{\prime}, 6$-diamidino-2phenylindole (Molecular Probes). Image analysis was performed using NIH ImageJ (public domain software, $\mathrm{NIH}$, Bethesda, MD, USA). SRY fluorescence was quantitated as described before. ${ }^{25}$ Briefly, measurements (average of two independent transfections, $n=50$ ) were taken of the density of fluorescence from the cytoplasm and the nucleus with the background fluorescence subtracted from the equation, Fn/c $=(n-b k g d n) /(c p-b k g d c p)$, where $n$ is the nucleus, bkgdn the background in the nucleus, $c p$ the cytoplasm and bkgdcp the background in the cytoplasm.

\section{Results}

\section{Clinical history}

A phenotypical female patient aged 26 years presented at the clinic with abdominal pain on the right side, which had previously been diagnosed as irritable bowel syn- drome. She had a history of treatment-resistant irregular menstrual cycles after menarche at the age of 14 years. Echographic examination revealed an enlarged ovary of $4.8 \times 3 \mathrm{~cm}$ on the right side, suspected to be a dermoid cyst. A right-sided salpingoophorectomy was performed by laparoscopy.

\section{Histological and immunohistochemical analyses}

Histological examination revealed that the removed lesion believed to be a dermoid cyst was in fact an exceptionally large $\mathrm{GB}$, of which a representative image of the hematoxylin and eosin (H\&E) staining is shown in Figure 1a. In agreement with this diagnosis, the germ cells showed positive staining for OCT3/4 (Figure 1b), TSPY (Figure 1c) and for SCF (Figure 1d). Furthermore, in the supportive cells, positive staining was observed for the granulosa cell marker, FOXL2 (Figure 1e), whereas SOX9 was absent (Figure 1f). Within the lesion, a small invasive component was identified by H\&E staining and immunohistochemistry, being a mixture of dysgerminoma, immature teratoma and yolk-sac tumor (data not shown). Tumor markers collected 26 days after removal of the tumor showed the following: AFP 5 (normal range: $0-9 \mu \mathrm{g} / \mathrm{l}$ ), hCG + beta hCG 2.0 (normal range: 0-6.9 IU/1) and LDH 391 (normal range: 0-449 U/1). Although the time delay between surgery and serum sampling for hormone level determination is considerable, it suggests that the tumor produced both AFP and hCG. On the basis of the decline of the markers and the (relatively small) invasive component situated in the middle of the tumor, the decision not to treat the patient with chemotherapy was taken, but surveillance was decided on. After 14 months, the patient was still relapse free, on the basis of tumor marker status, namely AFP 1, hCG + beta hCG $<0.1$ and LDH 219. The presence of a GB, staining positive for TSPY, initiated a discussion about the karyotype of the patient.

\section{In situ hybridization and karyotyping}

Fluorescent in situ hybridization was performed on the gonadal tissue, (see Figure 1g) and in parallel, karyotyping of peripheral lymphocytes was performed. Both approaches showed the presence of both a single $X$ and a single Y chromosome per nucleus.

\section{Prophylactically removed gonad}

After intensive discussion with the patient, the remaining left gonad was surgically removed, which on laparoscopical analysis presented as a streak gonad. This was consistent with the non-functionality of the in situ gonad, based on the patients' requirement for hormonal support to prevent menopause after the initial surgery. Histological examination revealed predominantly a streak appearance, with a small GB lesion, confirmed by a positive staining for OCT3/4, TSPY, SCF (data not shown) and FOXL2 (Figure $2 \mathrm{a}$ ). In contrast to the GB lesion in the right gonad, 

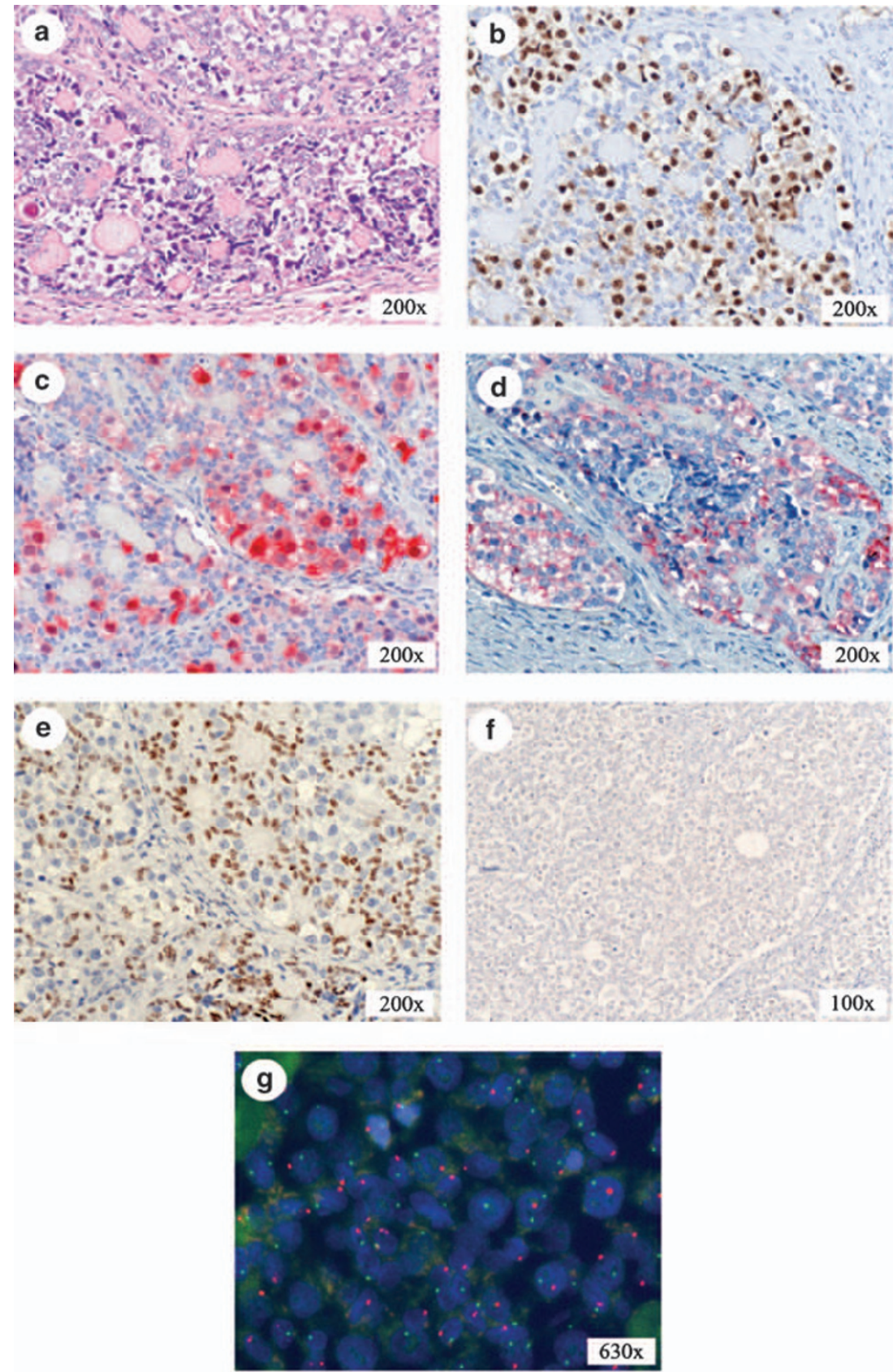

Figure 1 Immunohistochemical staining and fluorescent in situ hybridization of the right gonadoblastoma lesion. (a) Representative hematoxylin and eosin staining; The germ cells present in the GB stain positive for (b) OCT3/4, (c) TSPY and (d) SCF. (e) The supportive cells in the GB lesion are FOXL2 positive and (f) SOX9 negative. Magnification $100 \times$ and $200 \times$ for all. Slides (panels b-f) are counterstained with hematoxilin. (g) Representative FISH with Y centromere shown in red and X centomere shown in green. Magnification, $\times 630$.

SOX9-positive supportive cells were also identified (Figure 2b). Double staining for both FOXL2 and SOX9 showed that in the majority of cells, expression was mutually exclusive; however, a small subset of cells seemed to stain positive for both markers (Figure 2c and d).

\section{Mutational and functional analysis of SRY}

One of the first genes implicated in $46, \mathrm{XY}$ sex reversal is $S R Y$. A direct sequence analysis of the $S R Y$ gene showed the presence of a single nucleotide change at position 209 ( $G$ to $T$, see Figure 3a), resulting in a missense mutation (tryptophan (W) to leucine (L) amino acid change) at 

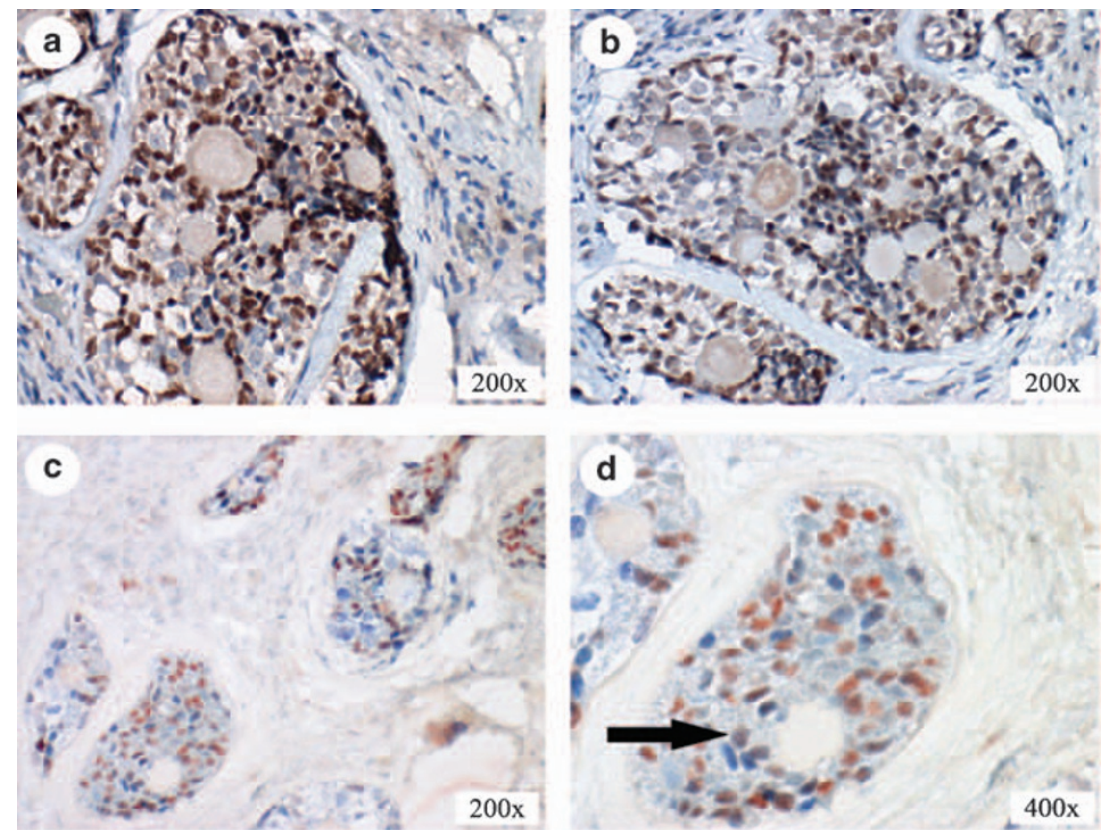

Figure 2 Immunohistochemical staining of the left gonadoblastoma lesion. (a) Supportive cells in the GB staining positive for FOXL2. (b) In addition, SOX9-positive supportive cells can be found. All slides are counterstained with hematoxylin. (c and d) Double staining for SOX9 (red) and FOXL2 (blue) of the left GB lesion. It must be noted that although most cells stain positive for either SOX9 or FOXL2, a few cells seem to have coexpression of both markers, indicated by an arrow. Magnification, $\times 200$ and $\times 400$ for all.

position 70 in the SRY protein. A W70L missense mutation in SRY has not been reported to date.

The W70L sequence variant is located within the $\mathrm{N}$ terminal NLS sequence of SRY (Figure 3b). Therefore, the nuclear import activity of this SRY mutant was investigated in a cell-based transfection assay using expression plasmids encoding wild-type and mutant full-length SRY. The subcellular localization of SRY was determined using indirect immunofluorescence. Wild-type SRY efficiently accumulated in the nucleus (Figure 3c, upper panel). As a positive control of a nuclear accumulation defect, the mutant R75N was included, showing a strong reduction of nuclear accumulation (28\% of wild type) consistent with a previous observation ${ }^{24}$ (Figure 3c, middle panel and Figure 3d). The mutant W70L also showed a significant reduction $(P<0.0001)$ of the nuclear accumulation to $\sim 50 \%$ of the wild-type SRY activity (Figure 3c lower panel and Figure 3d).

\section{Discussion}

GB is specifically found as a premalignant lesion in DSD patients with, among others, 46, XY gonadal dysgenesis (Swyer's syndrome) (see Cools et $a l^{2}$ for a review). The failure of the indifferent gonads to develop during embryogenesis into testes ultimately leads to a phenotypical female, who will present with delayed puberty and amenorrhea. Therefore, it is unusual to find GB in a presumed normal female after puberty, as is observed in the patient described in this study.

The GB in the right gonad was unusually large, and on sonographic investigation suspected for a dermoid cyst. The tumor cells were positive for the pluripotency marker, OCT3/4, which is specifically and consistently expressed in the neoplastic precursors of type II GCTs, ie, CIS in testis and GB in the dysgenetic gonad. ${ }^{14,16,26}$ The presence of this protein is essential for pluripotency of embryonic stem cells; and early in development, expression becomes confined to the germ cell lineage. Loss of OCT3/4 expression in mouse primordial germ cells leads to apoptosis, ${ }^{27}$ and thus is required for their survival. Upon differentiation of primordial germ cells/gonocytes to either pre-spermatogonia or oogonia, OCT3/4 is lost. Therefore, it is informative to identify embryonic germ cells, during normal development, as well as malignant germ cells.

As OCT3/4 cannot distinguish between malignant germ cells and germ cells with maturation delay, staining for SCF was performed. This marker has most recently been shown to specifically stain CIS and GB, whereas germ cells with proven maturation delay were negative. ${ }^{18}$ As expected, positive SCF staining was observed.

The presence of, part of, the $\mathrm{Y}$ chromosome is necessary for the development of a GB in a dysgenetic gonad. This is the so-called GB locus on the Y-chromosome (GBY) region as originally postulated by Page. ${ }^{19}$ This genomic region contains TSPY as one of the likely candidate genes. This is 
a
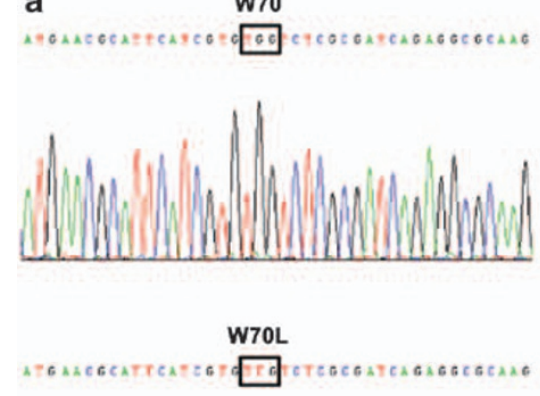

b

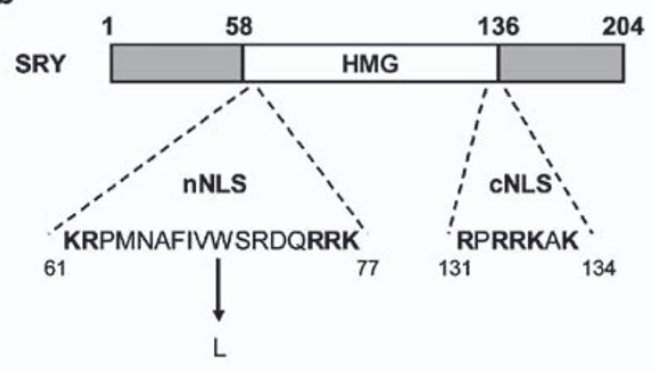

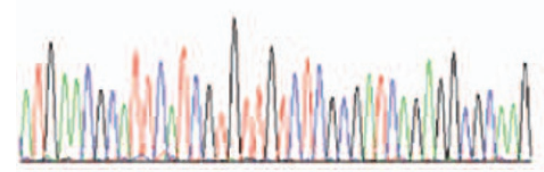
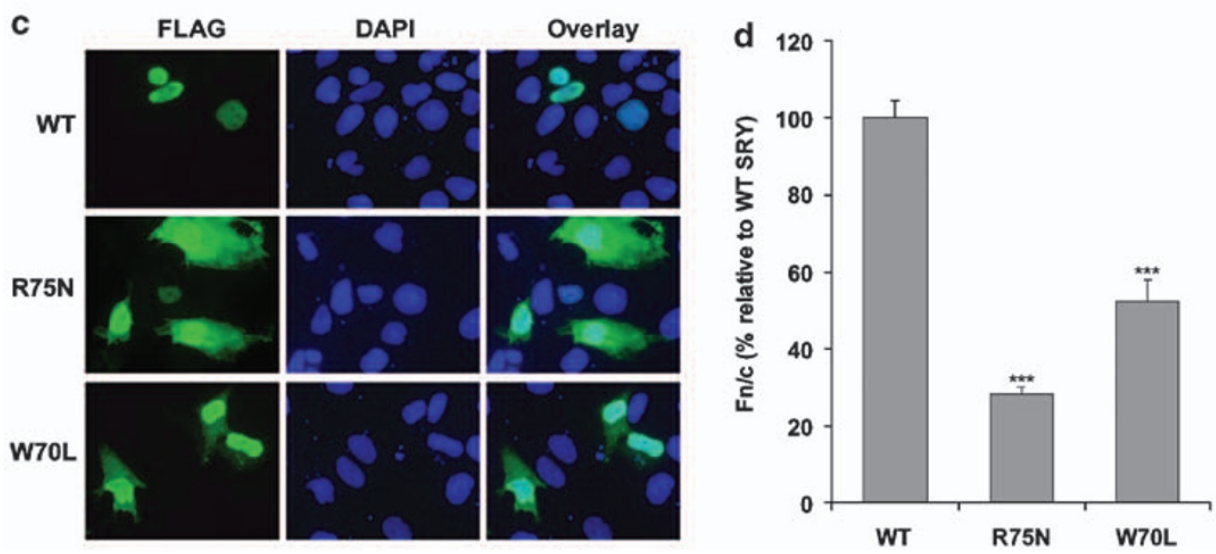

Figure 3 Mutational and functional analysis of SRY. (a) Wild type (WT, upper panel, control) and mutated sequence (lower panel, patient) of SRY. The G209T mutation results in a tryptophan (W) to leucine (L) amino acid change at position 70 of the SRY protein. (b) Schematic representation of the SRY protein; HMG domain and both the N- and C-terminal NLS are indicated. The W70L mutation resides in the N-terminal NLS. (c) Immunofluorescent analysis of WT and mutant FLAG tagged full-length SRY constructs (upper panel; nuclear accumulation of WT SRY, middle panel; nuclear accumulation of SRY R75N, and lower panel; nuclear accumulation of SRY W70L). The reduction in nuclear accumulation for both mutants compared with WT is noted. (d) SRY fluorescence was quantitated as described before. ${ }^{25}$ The SRY R75N control shows a strong reduction of nuclear accumulation $\left(28 \%\right.$ of WT), in line with previous observation. ${ }^{24}$ The mutant W70L SRY shows a significant reduction of nuclear accumulation of $\sim 50 \%$. Error bars represent the SE of mean values. A two-tailed $t$-test of unpaired sample means was performed, ${ }^{* \star *} P<0.0001$ for both.

supported by the strong expression of TSPY in CIS and the germ cells of $\mathrm{GB}^{28}$ as is also the case in this patient. Normally, TSPY is expressed in spermatogonia of the adult testis and is believed to be related to mitotic proliferation. $^{29}$ In fact, it influences cell-cycle-associated genes when transfected into NIH3T3 and HELA cells, ${ }^{30}$ as well as genes on chromosome $12 \mathrm{p}$, including KRAS2 and $N A$ NOG. ${ }^{31}$ This is an intriguing finding because the chromosomal constitution of CIS cells is overall similar to that of invasive type II GCTs, although gain of $12 p$ is established during progression to invasiveness. ${ }^{32,33}$

The SOX9 and FOXL2 staining patterns observed in the right GB lesion of this patient can be explained by the differentiation state of the gonad. In a XY individual, after SRY, the expression of SOX9 $9^{34}$ is necessary for testis development. SOX9 recruits a subset of cells in the indifferent gonad to the Sertoli cell fate and these orchestrate further testis formation and subsequent formation of the male phenotype. ${ }^{4,35}$ Activation of female gonadal development, which was initially believed to be the simple result of the absence of male development, requires activation of, among others, FOXL2. This transcription factor is required for the development of granulosa cells. ${ }^{36,37}$ The presence of SOX9 and FOXL2 can be used as a readout of testicular and ovarian differentiation, respectively. Moreover, stromal cells associated with GB are more granulosa-like cells, in contrast to CIS, which is associated with Sertoli cells. ${ }^{11}$ The absence of SOX9 expression in the right gonad of this patient has led to the development in a more female direction, ie, less male, as shown by the presence of FOXL2, which has led to the environment in which the GB has been able to 
develop. The expression pattern observed in the GB of the left gonad differs from that of our previous observations. ${ }^{11}$ Besides FOXL2-positive cells, some SOX9 staining, and even cells which seemed to stain positive for both was observed. In this context, it is of interest that XY Sox $9^{-1-}$ mice (in the presence of Sry) show expression of two ovarian genes (Wnt4 and Foxl2). ${ }^{38}$ This indicates that Sox9 can downregulate Wnt4 and/or Foxl2 in a XY gonad. In addition, evidence that Sertoli and granulosa cells have a common precursor exists. ${ }^{39}$ The recent report of a transcriptional (nuclear) function for SRY in the direct binding and regulation of the SOX9 gene ${ }^{5}$ increases the possibility that in this patient, reduced SOX9 levels can be an initial consequence of SRY. Overall 50\% of wild-type activity might place SRY at a threshold of activity, whereby in some cells sufficient levels of SOX9 are reached and so FOXL2 is downregulated, whereas in other cells this threshold is not reached, as shown by the FOXL2-positive and SOX9/FOXL2-double-positive cells. In mice, transfer of $Y$ chromosomes (SRY alleles) from certain strains onto the C57BL/6J mouse strain causes an abnormal gonadal development due to aberrant interaction with autosomal genes, leading to ovaries, ovotestis or delayed testis cord development in a XY background. ${ }^{40}$ Our study increases the possibility that other gonadal dysgenesis patients with GB and a similar FOXL2 and SOX9 staining pattern may be due to the $S R Y$ mutation causing partial loss of function.

The presence of a GB staining positive for TSPY warranted further investigation into the karyotype of this patient, which was shown to be $46, \mathrm{XY}$ by FISH and blood karyotyping. Subsequent laparoscopical examination and removal of the left gonad showed it to be a streak gonad containing a small GB lesion, staining positive for OCT3/4, TSPY, c-KIT and SCF as expected, which, if left in place, would eventually have developed into an invasive malignant tumor.

The presence of SOX9 in the GB of the left gonad shows that although nuclear import of SRY is impaired, it has been able, in a subset of cells, to induce expression of downstream targets and initiate the male pathway. Intriguing in this respect is the fact that, in contrast to most patients with Swyer's syndrome, the patient indicated that she had always been attracted to male activities, and considers herself to be bi-sexual. Sex-specific differences in brain morphology are driven by the action of steroid hormones produced by the gonads. Recent data suggest that also genetic differences and even SRY itself have a role. ${ }^{41-43}$ Possibly reduced penetrance of the mutation in brain sex development, either by some expression of steroid hormones by the gonads or directly because of SRY expression in the brain, has led to a more male development in this patient. Whether the $S R Y$ mutation has arisen de novo or is in the germ line cannot be determined as DNA from relevant family members is unavailable, although the first hypothesis is most likely.
Taken together, the reduced ability of the novel W7OL mutant SRY, as identified in this patient, to induce proper testis differentiation, reflected by the FOXL2 staining in the supportive cell lineages, created an environment in which germ cells were delayed in maturation. Prolonged expression of OCT3/4 and the presence of TSPY are suggested to allow subsequent survival and proliferation of these germ cells, which over time developed into a GB and invasive components. Knowledge about these issues will allow early (molecular) diagnosis of patients with a high risk for development of a malignant GCT, leading to prophylactic removal of the gonad and prevention of formation of an invasive cancer.

\section{Acknowledgements}

This work was financially supported by Translational Research Grant Erasmus MC 2006 (RH), Dutch Cancer Society Grant 2006-3607 (BdL) and the National Health and Medical Research Council Australia (VRH).

\section{Conflict of interest}

The authors declare no conflict of interest.

\section{References}

1 Hughes IA, Houk C, Ahmed SF, Lee PA, Group LC, Group EC: Consensus statement on management of intersex disorders. Arch Dis Child 2006; 91: 554-563.

2 Cools M, Drop SL, Wolffenbuttel KP, Oosterhuis JW, Looijenga LH: Germ cell tumors in the intersex gonad: old paths, new directions, moving frontiers. Endocr Rev 2006; 27: 468-484.

3 Sekido R, Bar I, Narvaez V, Penny G, Lovell-Badge R: SOX9 is upregulated by the transient expression of SRY specifically in Sertoli cell precursors. Dev Biol 2004; 274: 271-279.

4 Wilhelm D, Martinson F, Bradford S et al: Sertoli cell differentiation is induced both cell-autonomously and through prostaglandin signaling during mammalian sex determination. Dev Biol 2005; 287: $111-124$.

5 Sekido R, Lovell-Badge R: Sex determination involves synergistic action of SRY and SF1 on a specific Sox9 enhancer. Nature 2008; 453: 930-934.

6 Wilhelm D, Koopman P: The makings of maleness: towards an integrated view of male sexual development. Nat Rev Genet 2006; 7: $620-631$.

7 Harley VR, Lovell-Badge R, Goodfellow PN: Definition of a consensus DNA binding site for SRY. Nucleic Acids Res 1994; 22: $1500-1501$.

8 Giese K, Pagel J, Grosschedl R: Distinct DNA-binding properties of the high mobility group domain of murine and human SRY sexdetermining factors. Proc Natl Acad Sci USA 1994; 91: 3368-3372.

9 Scully RE: Gonadoblastoma/ a review of 74 cases. Cancer 1970; 25 : 1340-1356

10 Oosterhuis JW, Looijenga LH: Testicular germ-cell tumours in a broader perspective. Nat Rev Cancer 2005; 5: 210-222.

11 Hersmus R, Kalfa N, de Leeuw B et al: FOXL2 and SOX9 as parameters of female and male gonadal differentiation in patients with various forms of disorders of sex development (DSD). J Pathol 2008; 215: 31-38.

12 Almstrup K, Ottesen AM, Sonne SB et al: Genomic and gene expression signature of the pre-invasive testicular carcinoma in situ. Cell Tissue Res 2005.

13 Honecker F, Stoop H, de Krijger RR, Chris Lau YF, Bokemeyer C, Looijenga LH: Pathobiological implications of the expression of 
markers of testicular carcinoma in situ by fetal germ cells. J Pathol 2004; 203: 849-857.

14 Looijenga LH, Stoop H, de Leeuw HP et al: POU5F1 (OCT3/4) identifies cells with pluripotent potential in human germ cell tumors. Cancer Res 2003; 63: 2244-2250.

15 de Jong J, Stoop H, Dohle GR et al: Diagnostic value of OCT3/4 for pre-invasive and invasive testicular germ cell tumours. J Pathol 2005; 206: 242-249.

16 Cheng L, Sung MT, Cossu-Rocca P et al: OCT4: biological functions and clinical applications as a marker of germ cell neoplasia. J Pathol 2007; 211: 1-9.

17 Cools M, van Aerde K, Kersemaekers AM et al: Morphological and immunohistochemical differences between gonadal maturation delay and early germ cell neoplasia in patients with undervirilization syndromes. J Clin Endocrinol Metab 2005; 90: 5295-5303.

18 Stoop H, Honecker F, van de Geijn GJ et al: Stem cell factor as a novel diagnostic marker for early malignant germ cells. J Pathol 2008; 216: 43-54.

19 Page DC: Hypothesis: a Y-chromosomal gene causes gonadoblastoma in dysgenetic gonads. Development 1987; 101: 151-155.

20 Schnieders F, Dork T, Arnemann J, Vogel T, Werner M, Schmidtke $\mathrm{J}$ : Testis-specific protein, Y-encoded (TSPY) expression in testicular tissues. Hum Mol Genet 1996; 5: 1801-1807.

21 Lau Y, Chou P, Iezzoni J, Alonzo J, Komuves L: Expression of a candidate gene for the gonadoblastoma locus in gonadoblastoma and testicular seminoma. Cytogenet Cell Genet 2000; 91: 160-164.

22 Stoop H, Honecker F, Cools M, de Krijger R, Bokemeyer C, Looijenga LH: Differentiation and development of human female germ cells during prenatal gonadogenesis: an immunohistochemical study. Hum Reprod 2005; 20: 1466-1476.

23 Kido T, Lau YF: A Cre gene directed by a human TSPY promoter is specific for germ cells and neurons. Genesis 2005; 42: 263-275.

24 Harley VR, Layfield S, Mitchell CL et al: Defective importin beta recognition and nuclear import of the sex-determining factor SRY are associated with XY sex-reversing mutations. Proc Natl Acad Sci USA 2003; 100: 7045-7050.

25 Argentaro A, Sim H, Kelly S et al: A SOX9 defect of calmodulindependent nuclear import in campomelic dysplasia/autosomal sex reversal. J Biol Chem 2003; 278: 33839-33847.

26 De Jong J, Stoop J, Dohle GR et al: Diagnostic value of OCT3/4 for pre-invasive and invasive testicular germ cell tumors. J Pathol 2005; 206: 242-249.

27 Kehler J, Tolkunova E, Koschorz B et al: Oct4 is required for primordial germ cell survival. EMBO Rep 2004; 5: 1078-1083.

28 Kersemaekers AM, Honecker F, Stoop $\mathrm{H}$ et al: Identification of germ cells at risk for neoplastic transformation in gonadoblastoma: an immunohistochemical study for OCT3/4 and TSPY. Hum Pathol 2005; 36: 512-521.
29 Lau YF: Gonadoblastoma, testicular and prostate cancers, and the TSPY gene. Am J Hum Genet 1999; 64: 921-927.

30 Oram SW, Liu XX, Lee TL, Chan WY, Lau YF: TSPY potentiates cell proliferation and tumorigenesis by promoting cell cycle progression in HeLa and NIH3T3 cells. BMC Cancer 2006; 6: 154.

31 Li Y, Tabatabai ZL, Lee TL et al: The Y-encoded TSPY protein: a significant marker potentially plays a role in the pathogenesis of testicular germ cell tumors. Hum Pathol 2007; 38: 1470-1481.

32 Rosenberg C, Van Gurp RJ, Geelen E, Oosterhuis JW, Looijenga LH: Overrepresentation of the short arm of chromosome 12 is related to invasive growth of human testicular seminomas and nonseminomas. Oncogene 2000; 19: 5858-5862.

33 Summersgill B, Osin P, Lu YJ, Huddart R, Shipley J: Chromosomal imbalances associated with carcinoma in situ and associated testicular germ cell tumours of adolescents and adults. Br J Cancer 2001; 85: 213-220.

34 Foster JW, Dominguez-Steglich MA, Guioli S et al: Campomelic dysplasia and autosomal sex reversal caused by mutations in an SRY-related gene. Nature 1994; 372: 525-530.

35 Morais da Silva S, Hacker A, Harley V, Goodfellow P, Swain A, Lovell-Badge R: Sox9 expression during gonadal development implies a conserved role for the gene in testis differentiation in mammals and birds. Nat Genet 1996; 14: 62-68.

36 Schmidt D, Ovitt CE, Anlag $\mathrm{K}$ et al: The murine winged-helix transcription factor Foxl2 is required for granulosa cell differentiation and ovary maintenance. Development 2004; 131: 933-942.

37 Uda M, Ottolenghi C, Crisponi L et al: Foxl2 disruption causes mouse ovarian failure by pervasive blockage of follicle development. Hum Mol Genet 2004; 13: 1171-1181.

38 Barrionuevo F, Bagheri-Fam S, Klattig J et al: Homozygous inactivation of Sox9 causes complete XY sex reversal in mice. Biol Reprod 2006; 74: 195-201.

39 Albrecht KH, Eicher EM: Evidence that Sry is expressed in pre-Sertoli cells and Sertoli and granulosa cells have a common precursor. Dev Biol 2001; 240: 92-107.

40 Albrecht KH, Young M, Washburn LL, Eicher EM: Sry expression level and protein isoform differences play a role in abnormal testis development in C57BL/6J mice carrying certain Sry alleles. Genetics 2003; 164: 277-288.

41 Dewing P, Shi T, Horvath S, Vilain E: Sexually dimorphic gene expression in mouse brain precedes gonadal differentiation. Brain Res Mol Brain Res 2003; 118: 82-90.

42 De Vries GJ, Rissman EF, Simerly RB et al: A model system for study of sex chromosome effects on sexually dimorphic neural and behavioral traits. J Neurosci 2002; 22: 9005-9014.

43 Dewing P, Chiang CW, Sinchak K et al: Direct regulation of adult brain function by the male-specific factor SRY. Curr Biol 2006; 16: $415-420$. 